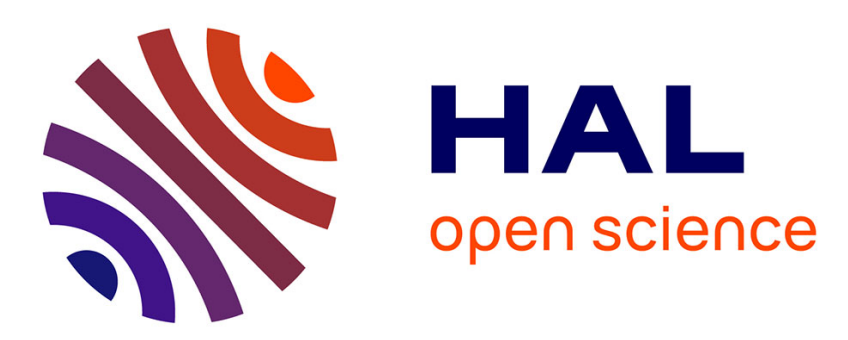

\title{
Le baromètre de l'âme : émotions et couleurs du temps dans l'œuvre de jeunesse de Thomas Mann (1893-1912)
}

Sylvie Jacqueline Grimm-Hamen

\section{To cite this version:}

Sylvie Jacqueline Grimm-Hamen. Le baromètre de l'âme : émotions et couleurs du temps dans l'œuvre de jeunesse de Thomas Mann (1893-1912). Germanica, 2017, 60, pp.31-45. 10.4000/germanica.3645 . hal-02559267

\section{HAL Id: hal-02559267 \\ https://hal.science/hal-02559267}

Submitted on 22 Sep 2020

HAL is a multi-disciplinary open access archive for the deposit and dissemination of scientific research documents, whether they are published or not. The documents may come from teaching and research institutions in France or abroad, or from public or private research centers.
L'archive ouverte pluridisciplinaire HAL, est destinée au dépôt et à la diffusion de documents scientifiques de niveau recherche, publiés ou non, émanant des établissements d'enseignement et de recherche français ou étrangers, des laboratoires publics ou privés. 


\title{
LE BAROMĖTRE DE L'ÂME : ÉMOTIONS ET COULEURS DU TEMPS DANS L'EUVRE DE JEUNESSE DE THOMAS MANN (1893-1912)
}

\author{
Sylvie Grimm-Hamen
}

Université de Lille | « Germanica»

$2017 / 1 n^{\circ} 60 \mid$ pages 31 à 45

ISSN 0984-2632

Article disponible en ligne à l'adresse :

https://www.cairn.info/revue-germanica-2017-1-page-31.htm

Distribution électronique Cairn.info pour Université de Lille.

(C) Université de Lille. Tous droits réservés pour tous pays.

La reproduction ou représentation de cet article, notamment par photocopie, n'est autorisée que dans les limites des conditions générales d'utilisation du site ou, le cas échéant, des conditions générales de la licence souscrite par votre établissement. Toute autre reproduction ou représentation, en tout ou partie, sous quelque forme et de quelque manière que ce soit, est interdite sauf accord préalable et écrit de l'éditeur, en dehors des cas prévus par la législation en vigueur en France. Il est précisé que son stockage dans une base de données est également interdit. 


\section{Germanica}

\section{Le baromètre de l'âme : émotions et couleurs du temps dans l'œuvre de jeunesse de Thomas Mann (1893-1912)}

Das Seelenbarometer : Emotionen und Wetterlage in Thomas Manns Frühwerk (1893-1912)

The barometer of the soul: emotions and weather conditions in Thomas Mann's early short stories (1893-1912)

\section{Sylvie Grimm-Hamen}

\section{grevues.org}

Édition électronique

URL : http://germanica.revues.org/3645

DOI : 10.4000/germanica.3645

ISSN : 2107-0784

\section{Éditeur}

CeGes Université Charles-de-Gaulle Lille-III

\section{Édition imprimée}

Date de publication : 30 juin 2017

Pagination : 31-45

ISBN : 9782913857391

ISSN : 0984-2632

Distribution électronique Cairn

CAIRN

CHERCHER, REPÉRER, AVANCER.

Référence électronique

Sylvie Grimm-Hamen, « Le baromètre de l'âme : émotions et couleurs du temps dans l'œuvre de jeunesse de Thomas Mann (1893-1912) », Germanica [En ligne], 60 | 2017, mis en ligne le 30 juin 2019, consulté le 27 juillet 2017. URL : http://germanica.revues.org/3645 ; DOI : 10.4000/germanica.3645 


\title{
Le baromètre de l'âme : émotions et couleurs du temps dans l'œuvre de jeunesse de Thomas Mann (1893-1912)
}

\author{
Sylvie GRIMM-HAMEN \\ Université de Lorraine (Nancy)
}

Les « états d'âme » ${ }^{1}$ des personnages mis en scène par Thomas Mann ne sont pas séparables de l'état du ciel et du temps qu'il fait. La montée des températures, les mouvements du vent, le rythme des saisons et les sons ou les odeurs qu'ils charrient sont souvent discrètement, mais constamment présents. Ils n'accompagnent pas seulement la montée des désirs, le dessin des rêves, l'éveil des sentiments, les rouages de la pensée. Ils sont aussi vécus et subis, parfois implacablement. L'humeur peut varier du tout au tout avec l'obscurcissement de l'horizon, le rougeoiment du crépuscule, l'arrivée d'un orage. Les effets, tant physiologiques que psychologiques, de cette sensibilité climatique peuvent paraître mineurs au regard du pouvoir que la musique peut exercer sur eux, ils n'en participent pas moins à leur sentiment d'existence.

1. - En français dans le texte. Toutes les références concernent l'édition suivante : Thomas Mann, Frühe Erzählungen 1893-1912. In der Fassung der Großen kommentierten Frankfurter Ausgabe, Frankfurt a.M, S. Fischer Verlag, 2008, 2014, p. 29. Elles seront indiquées entre parenthèses dans le texte. 
Écoutons le Paillasse dire l'expérience euphorisante que lui procurent les vibrations chaudes de l'air ou la douceur suave d'une brise qui entrent par sa fenêtre :

[...] mais un matin, au réveil, tu remarques, incrédule, qu'un rai de lumière bleu, lumineux, perce les rideaux de ta chambre. Tout surpris, tu sautes du lit, tu ouvres la fenêtre, un flot de lumière frémissante t'inonde [...] et en même temps tu perçois, à travers le vacarme de la rue, le gazouillis incessant et joyeux des oiseaux, et tu as le sentiment que tu inspires avec l'air frais et léger d'un premier jour d'octobre, l'arôme incomparablement doux et prometteur, dont sont porteurs d'habitude les vents de mai. C'est le printemps [...] et tu sautes dans tes vêtements pour sortir au plus vite à l'air libre et parcourir les rues sous un ciel chatoyant...2

Ou, à l'inverse, l'inconnu de Jena (Schiller, en l'occurrence) déplorer dans Schwere Stunde (1905) l'effet du mauvais temps sur l'état de ses nerfs $^{3}$ :

[...] il est vrai que le mauvais temps régnait à Iéna depuis des semaines et des semaines, un temps misérable, exécrable, que l'on sentait dans tous ses nerfs, rude, sombre et froid; et les hurlements du vent de décembre dans le conduit du poêle avaient quelque chose de si désolé de Dieu, de si maudit, que l'on songeait à une lande dans la nuit, sous la tempête, et aux errements d'une âme en peine ${ }^{4}$.

Vivre pour Paolo Hofmann dans Der Wille zum Glück (1896) ou Gustav Aschenbach dans Der Tod in Venedig (1912) revient à quitter la grisaille brumeuse du Nord pour rejoindre l'intensité solaire du Sud.

2. - « [...] eines Morgens aber beim Erwachen bemerkst du mit ungläubigen Augen, daß ein schmaler Streif von leuchtendem Blau zwischen den Fenstervorhängen hindurch in dein Zimmer blitzt. Ganz erstaunt springst du aus dem Bett, du öffnest das Fenster, eine Woge von zitterndem Sonnenlicht strömt dir entgegen, und zugleich vernimmst du durch alles Straßengeräusch hindurch ein geschwätziges und munteres Vogelgezwitscher, während es dir nicht anders ist, als atmetest du mit der frischen und leichten Luft eines ersten Oktobertages die unvergleichlich süße und verheißungsvolle Würze ein, die sonst den Winden des Mai gehört. Es ist Frühling [...] und du wirfst dich in die Kleider, um unter dem schimmernden Himmel durch die Straßen und ins Freie zu eilen. » (144).

3. - Les nerfs deviennent à cette époque les organes de la sensibilité. Cf. Hermann Bahr : «Der neue Idealismus drückt die neuen Menschen aus. Sie sind Nerven [...]. Sie erleben nur mehr mit den Nerven, sie reagieren nur mehr von den Nerven aus. » Hermann, Bahr : «Die Überwindung des Naturalismus », in : Gotthart Wunberg (dir.), Die Wiener Moderne. Literatur, Kunst und Musik zwischen 1890 und 1910, Stuttgart, Reclam, 1981, 1992, p. 204.

4. - « [...] und schlechtes Wetter war über Jena, seit Wochen, das war richtig, ein miserables und hassenswertes Wetter, das man in allen Nerven spürte, wüst, finster und kalt, und der Dezemberwind heulte im Ofenrohr, verwahrlost und gottverlassen, daß es klang nach nächtiger Heide im Sturm und Irrsal und heillosem Gram der Seele. » (419). 
Pour autant, les récits ne se laissent jamais réduire à des antagonismes triviaux entre lumières bienfaisantes et ténèbres délétères. De même qu'il y a un Sud géographique et existentiel qui vivifie, qui intensifie l'existence, aiguise les sens comme les idées, il y a aussi la chaleur brûlante et les exhalaisons d'une terre surchauffée qui anéantissent toute velléité d'action, qui laissent les corps apathiques, en sueur, incapables de penser. Quand souffle le sirocco, en provenance du désert, toute énergie se défait, plus rien n'est possible. Ce vent-là rend Gustav Aschenbach incapable de sentir, de vouloir, de créer (587). Il anesthésie et paralyse. Il amollit, dévitalise, désorganise. Au lieu de devenir plus intensément lui-même dans ces régions du Sud dont il attendait « l'épanouissement de son art » (264), Tonio Kröger s'y trouve coupé de ce qu'il est, exilé de lui-même. Ce n'est que sous l'effet des bourrasques du vent du Nord, dans les rafales au «parfum âcre » (299) que son cœur et son esprit sortent de leur «pétrification » $(315)^{5}$.

Ces liens que les phénomènes atmosphériques ${ }^{6}$ entretiennent avec les aléas du moi s'inscrivent dans l'exacerbation d'un subjectivisme caractéristique de la modernité littéraire autour de 1900. Ils portent le sceau des théoriciens et des écrivains de la décadence, Paul Bourget et Hermann Bahr, qui entendent « dépasser le naturalisme » et sa philosophie positiviste en cultivant les nuances d'atmosphère dans lesquelles se miroite l'état émotionnel du sujet. Ils se situent également dans l'horizon épistémologique de la philosophie schopenhauerienne et de son présupposé métaphysique concernant l'unité intime des phénomènes naturels et spirituels ${ }^{7}$. Les effets des « caprices du mercure », auxquels Thomas Mann consacre un chapitre entier dans Der Zauberberg (1924), en référence au Dieu Hermès, n'ont toutefois guère été pris en considération par la critique dans les premiers récits de l'écrivain ${ }^{8}$. Astrid Roffmann tend

5. - Dans son discours de remerciement pour l'obtention du prix Nobel en 1929, l'écrivain évoque la nouvelle en ces termes : « Sie handelt vom Süden und vom Norden und von der Mischung beider in einer Person [...]. Der Süden, das ist in dieser Geschichte der Inbegriff alles geistig-sinnlichen Abenteuers, der kalten Leidenschaft des Künstlertums; der Norden dagegen der Inbegriff aller Herzlichkeit und bürgerlichen Heimat, alles tief ruhenden Gefühls, aller innigen Menschlichkeit. » In : Gesammelte Werke in zwölf (dreizehn) Bänden, Frankfurt a.M., Fischer, 1960, 1974, Bd. XI, 410 [Désormais: $G W]$.

6. - Nous aurons ici une acception large du terme en désignant par là tous les phénomènes observés dans l'atmosphère et les manifestations qui s'y rattachent: pluie, neige, vent, soleil, mais aussi températures et saisons.

7. - Cf. Børge Kristiansen, « Das Problem des Realismus bei Thomas Mann. Leitmotiv-Zitat-Mythische Wiederholungsstruktur », in : Helmut Koopmann (dir.), Thomas Mann Handbuch, Frankfurt a.M., Fischer Taschenbuch Verlag, 2005 [2001], p. 824 sqq.

8. - Wolfgang Schneider consacre un article au motif du froid dans le second Thomas Mann Handbuch édité en 2015. Dans ce contexte, il n'évoque que les romans de l'écrivain. Cf. Wolfgang Schneider, «Kälte », in : Andreas Blödorn, Friedhelm Marx 
à considérer, par exemple, que, dans les œuvres de jeunesse, la météorologie reste subordonnée au thème « majeur» de la tension entre l'art et la vie. Appréhendés comme les objets d'une allégorisation qui n'échappe pas toujours aux stéréotypes, l'état du ciel et les éléments naturels apparaissent essentiellement dès lors comme des surfaces de projection dont la fonction dramatique est de mettre en scène la sensibilité artistique des personnages ou leur ébranlement intérieur.

Dans le cadre restreint de cette étude, nous porterons notre attention sur quelques récits de jeunesse de Thomas Mann, et tenterons une évaluation quelque peu différente des évocations météorologiques qui les parsèment, en les envisageant dans leur rapport à l'émergence d'une nouvelle forme de sensibilité. Écrits entre 1893 et 1912, ces premiers textes constituent, en effet, des observatoires privilégiés de l'approfondissement progressif de l'appréciation de soi et du monde par les personnages. Corrélés au travail d'intériorisation du sujet et à la pratique de l'écriture de soi, l'« état du baromètre » (303) et, par métonymie, les conditions atmosphériques dans leur ensemble, disent quelque chose du rapport de ces derniers au présent, de l'expérience du corps et des imaginaires associés au temps qu'il fait. Dans une œuvre, en outre, où, aux dires de l'écrivain lui-même, "les descriptions de paysages $»^{9}$ le cèdent à « l'approfondissement subjectif des images de la réalité »10, les couleurs de l'air sont aussi un instrument permettant à l'auteur de sonder et de déjouer les représentations que les personnages se font de la réalité, et donc des moyens efficaces de subversion du réalisme.

*

Dans l'une des premières nouvelles de l'écrivain, Gefallen (1894), « une histoire d'amour qui mêle cynisme et sentimentalisme »11, selon Thomas Mann, et dont la critique a souvent condamné le «symbolisme

(dir.), Thomas Mann Handbuch.Leben - Werk - Wirkung, Stuttgart, J.B. Metzler, 2015, p. 310-311. Le froid est par ailleurs le seul motif «atmosphérique » analysé. Astrid Roffmann consacre, pour sa part, un chapitre entier de son travail au motif des intempéries, mais elle se concentre, elle aussi, sur les romans et les œuvres de la maturité. Parmi les nouvelles de jeunesse qu'elle analyse, dans le cadre plus large toutefois du traitement de la nature, se trouvent Gladius Dei (1902) et Der Tod in Venedig (1912). Des remarques éparses concernent aussi Gefallen. Cf. Astrid Roffmann, « Keine freie Note mehr. » Natur im Werk Thomas Manns, Würzburg, Königshausen und Neumann, 2003.

9. - « Lübeck als geistige Lebensform » (1926), GW, Bd. XI, p. 394. Cf. aussi p. 388 : «Es ist oft kritisch bemerkt und mir angekreidet worden, daß die Landschaftsschilderung nicht meine Stärke ist, daß sie zu kurz kommt in meiner Produktion. »

10. - Bilse und Ich (1906), GW, Bd. X, p. 16.

11. - Wilhelm Zils (dir.), Geistiges und künstlerisches München in Selbstbiographien, München, Max Kellerers Verlag, 1913, p. 231. 
plat $»^{12}$ ou le $«$ conventionnalisme $»^{13}$, les variations du temps, le rythme des jours et des saisons scandent l'histoire malheureuse de la passion du jeune docteur Selten pour la comédienne Irma Weltner. Ainsi, l'arrivée du printemps coïncide avec le début de leur relation amoureuse, narrée dans le récit enchâssé. Un ciel dégagé et lumineux, le gazouillis des oiseaux, le parfum des fleurs et l'air doux et frémissant du printemps, les rayons revigorants du soleil allégorisent, de même, le bonheur de l'étudiant et le revirement de son humeur (22).

Cet accord entre les émotions et le temps qu'il fait est souligné par le narrateur, non sans une certaine ambiguilté:

[...] alors que, jusqu'ici, il avait eu le sentiment de vivre sous pression, sous le poids d'une nécessité pesante, il agissait désormais selon sa volonté propre, avec détermination et joie, comme en état d'hypnose.

Mais c'était le printemps!14

Effrayé par sa soudaine témérité à vouloir déclarer sa flamme à la femme dont il est tombé amoureux, l'étudiant en médecine, dont on découvre qu'il s'agit du narrateur dans ses jeunes années, met son mépris des conventions sociales sur le compte des effets stimulants du printemps. Formulée sur le mode du discours indirect libre, sa remarque, « Mais c'était le printemps ! » vient justifier son attitude et livrer une explication qui s'impose à lui comme une évidence. La mise en exergue du propos pourrait toutefois suggérer aussi qu'il s'agit là de l'exclamation amusée du narrateur qui, avec le recul de l'âge, brocarde cette idiosyncrasie et la fougue d'un jeune homme confondant l'état du ciel et son humeur du moment.

Dans l'écriture, cette concordance entre le dedans et le dehors se traduit par l'anthropomorphisation de la nature et la cosmisation des émotions. Alors que «[t]ous les sens [du jeune homme] sont printaniers » (22), la nature, elle, est animée des mêmes pulsions que le corps, tenaillée par la même « soif » (31). De même, lorsque celui-ci cède aux larmes, «tout [est] triste. Le monde [est] si silencieux et assoiffé, et la lune si pâle $»^{15}$.

12. - Dieter Wolfgang Adolphs, Literarischer Erfahrungshorizont. Aufbau und Entwicklung der Erzählperspektive im Werke Thomas Manns, Heidelberg, Winter Verlag, 1985, p. 170.

13. - Peter Sprengel, Geschichte der deutschsprachigen Literatur 1900-1918. Von der Reichsgründung bis zur Jahrhundertwende, München, Verlag C.H. Beck, 1998, p. 399.

14. - « [...] während er bislang wie unter einem Druck gestanden, unter einer lastenden Notwendigkeit, wie in der Hypnose handelte er nun mit freiem, zielsicherem, jauchzendem Willen.

Es war ja Frühling! » (23).

15. - Alle Sinne waren ganz Frühling (22); « eine Befriedigung [...] all dieses 
Corrélée à la joie de vivre du jeune amoureux, l'appréciation du printemps est tributaire ici d'une rhétorique amoureuse que le narrateur dénoncera plus tard, à la fin du récit-cadre. Les odeurs et les atmosphères convoquées dans l'histoire d'amour ressortissent, de fait, à des poncifs en vogue au XIX ${ }^{e}$ siècle. Volker Mergenthaler a montré, par exemple, que dans l'iconographie de cette période, la femme aimée était régulièrement associée au printemps et au lilas ${ }^{16}$. D'un point de vue narratif, le « parallélisme » entre le temps qu'il fait et les sentiments éprouvés par le personnage relève, de même, d'une convention d'écriture ${ }^{17}$. Dans la littérature réaliste de l'époque, les saisons étaient traditionnellement assujetties à l'action proprement dite, et l'une des fonctions dramatiques qui leur étaient dévolues était d'accompagner, voire d'anticiper, les péripéties du sujet ${ }^{18}$.

La reprise, parfois terme à terme, dans les récits ultérieurs des mêmes "ingrédients atmosphériques ", associés aux mêmes humeurs, contribuent encore davantage à mettre en exergue le côté construit de cette représentation du printemps. Dans Der kleine Herr Friedemann, comme dans Luischen ou Tristan, les signes manifestes du printemps sont, comme ici, le « gazouillis des oiseaux » $(36,91,105,112,267)$, un « ciel bleu lumineux » $(22,35,70,105,267)$, la suave douceur de l'air $(22,88)$ et un soleil rayonnant. S'y rajoutent des odeurs et des conditions atmosphériques qui s'imposeront dans de nombreux récits comme les éléments obligés d'une " météorologie passionnelle » figurant l'ardeur des désirs et de la pulsion érotique, tels le parfum entêtant du jasmin dans Gefallen et Der kleine Herr Friedemann, le mélange dionysiaque d'humidité et de chaleur, un temps électrique et orageux dans Der Tod in Venedig. L'effet de répétition minore la portée référentielle de ces notations atmosphériques et les impose comme des leitmotive partici-

Durstes in ihm und der Natur» (31) ; « Es war alles so traurig. Die Welt war so stumm und durstig, und der Mond war so blaß. » (32).

16. - Volker Mergenthaler, « Der eigentliche Einsatz dieser mächtigen Schriftstellerschaft », in : Michael Ansel, Hans-Edwin Friedrich, Gerhard Lauer (dir.), Die Erfindung des Schriftstellers Thomas Mann, Berlin, De Gruyter, 2009, p. 173. L'auteur cite notamment l'exemple des tableaux de Pierre-Auguste Renoir : Femme auxlilas, 1877 ; de Marie Bashkirtseff : Jeune femme au bouquet de lilas, vers 1881 ; Sophie Anderson : Girl with lilaes, vers 1890. Willy Schumann suppose, pour sa part, que Thomas Mann aurait trouvé l'inspiration du motif chez Theodorm Storm. Cf. Willy Schumann, «Theodor Storm und Thomas Mann: Gemeinsames und Unterschiedliches », Monatshefte, Jg. 55, Madison: University of Wisconsin, 1963, p. 49-68.

17. - Eberhard Lämmert, Bauformen des Erzählens, Stuttgart, Metzler Verlag, 1989 [8e édition], p. 91. Cf. aussi Astrid Roffmann qui analyse ces parallélismes dans Buddenbrooks et souligne que ceux-ci sont très fréquents dans l'œuvre de jeunesse de Thomas Mann. A. Roffmann, « Keine freie Note mehr. », op. cit., p. 33.

18. - Friedrich Christian Delius, Der Held und sein Wetter. Ein Kunstmittel und sein ideologischer Gebrauch im Roman des bürgerlichen Realismus, München, Wallstein Verlag, 1971, p. 101. 
pant d'une scénographie des émotions. Ils se trouvent «englobés », de la sorte, dans un réseau de significations plus vaste et se trouvent soumis à des modèles interprétatifs dépassant leur valeur singulière dans chaque récit ${ }^{19}$. Peu importe, dans ce contexte, que le pied de lilas, présenté ironiquement par le narrateur comme un ressort éminent de l'intrigue (48), fleurisse de mai à la mi-juillet et soit une « aberration » d'un point de vue botanique 20 ! Il fait partie intégrante d'une mythologie de la lumière et des saisons qui privilégie le jeu avec les symboles à la vraisemblance et à l'ancrage dans le réel.

L'intérêt de cette nouvelle pour notre propos est toutefois que cet accord entre l'état du ciel et celui du sujet ne perdure pas. Arrivé au terme de son histoire, le narrateur persifle le fait que le jeune étudiant qu'il fut « s'en est laissé conter par l'odeur agréable et délicate du lilas $»^{21}$. Après avoir posé une sorte de fond de tableau idyllique et bucolique, celui-ci démasque les réflexes de l'imagination passionnée du jeune homme qui a pris ses désirs pour la réalité. Tandis que « dehors s'épanouit l'éclat majestueux d'une agréable journée d'été $»^{22}$, la catastrophe amoureuse, elle, se déploie. Le hiatus entre les valeurs émotionnelles associées au printemps (joie, bonheur, désir amoureux) et l'expérience vécue par le personnage devient patent. Le ciel toujours bleu, le gazouillement continu des oiseaux, les vapeurs du printemps apparaissent dès lors comme des clichés de ses débordements lyriques, et comme les «accessoires scéniques » (41) d'une imagerie du bonheur avec « voûte de nuages roses et putti rococo » (37). L'éclat du ciel rend manifeste l'aveuglement du personnage et son incapacité à comprendre la véritable condition d'Irma. Le printemps devient une allégorie de sa naïveté, en même temps que le signe stéréotypé d'un bonheur illusoire. Rendu «nerveux » par le printemps, Tonio Kröger dira plus tard, comme Adalbert, son embarras par rapport « aux souvenirs et sentiments triviaux qu'il génère » (269-270) et en fera une figure allégorique de sa vision ingénue de l'art $(265,271)$. L'exaltation suscitée par la venue du printemps contrevient à ses yeux au geste créateur, précisément parce que l'artiste, vaincu par ses émotions, manque du détachement, voire de la froideur nécessaires à l'accomplissement du travail artistique.

Ainsi, la voix discordante du narrateur du récit-cadre met en exergue, pour finir, le « silence » d'une nature foncièrement « indifférente »

19. - Cf. Andreas Blödorn, « Realismus », in : A. Blödorn, F. Marx (dir.), ThomasMann-Handbuch, op. cit., p. 268.

20. - Bernd Hamacher, « Poetologische Funktionen des Märchens », in : Thomas Eicher (dir.), Märchen und Moderne: Fallbeispiele einer intertextuellen Relation, Münster, LIT Verlag, 1996, p. 87.

21. - «Als er sich von dem lieben, weichen Duft genug hatte erzählen und verheißen lassen, ging er hinauf... » (41).

22. - «Draußen herrschte in lichter Majestät der liebliche Sommertag. » (47). 
(111) « qui feint de nous comprendre » (41) mais qui se prête mal aux projections auxquelles on la soumet, car elle a ses propres lois où l'hiver finit toujours par revenir (48). À la fin, c'est vers cette illusion d'une nature mimétique qu'il tourne sa colère et sa frustration, en brisant le pied de lilas «à l'odeur trompeuse » (47). On retrouve, du reste, une mise en abyme comparable dans Der Weg zum Friedhof, lorsque le narrateur ouvre son récit sur un tableau bucolique et l'image artificielle d'" un ciel divin bleu, parsemé de plein de petits morceaux de nuage ronds et compacts, tavelé de petits grumeaux d'un blanc neigeux à l'effet humoristique $»^{23}$, en inadéquation totale avec les sentiments du personnage. Ce qu'il relève d'ailleurs lui-même, non sans ironie : « Lobgott Piepsam n'était pas d'apparence joyeuse et son allure convenait mal à cette douce matinée ; même pour quelqu'un qui se rendait sur la tombe d'êtres chers, elle était bien trop triste $»^{24}$.

Ce découplage des saisons et des humeurs est un principe narratif dont l'écrivain usera abondamment par la suite pour dénoncer les illusions dont sont victimes ses personnages. On peut observer au demeurant que, dans ces premiers récits, le printemps incite la plupart d'entre eux à prendre des initiatives qui s'avèreront funestes. C'est dans l'éclosion du printemps que Friedemann puisera d'abord le courage de rendre visite à Gerda (105) avant que, lors de leur dernière rencontre, la douceur de l'air et de la lumière, la senteur des fleurs, le chant des cigales forment un contraste ironique avec la férocité des propos de Gerda et son propre abattement (116). Que Friedemann se tue par une nuit chaude et étoilée fait du printemps et de l'été les symboles funestes du malheur de l'existence et de ses mirages. (117) Les patients du sanatorium d'Einfried entreprennent, eux aussi, leur partie de luge sous l'effet d'un temps ensoleillé, «plus pur et lumineux que les jours précédents » (343) qui s'avèrera fatal à Frau Klöterjahn, restée seule avec Spinell. C'est encore le retour du printemps qui incite Amra, dans Luischen, à vouloir organiser la fête à laquelle succombera finalement son époux (166). On retrouve dans ces décalages l'empreinte de la philosophie schopenhauerienne, dénonciatrice des effets illusoires du monde phénoménal, en même temps que les effets d'un esthétisme de la décadence qui prend à rebours la hiérarchie des valeurs héritées du romantisme en subvertissant l'accord entre les variations du moi et celles du temps.

23. - «Gottes blauer Himmel war mit lauter kleinen, runden kompakten Wolkenstückchen besetzt, betupft mit lauter schneeweißen Klümpchen von humoristischem Ausdruck. » (211).

24. - «Lobgott Piepsams Erscheinung war nicht freudig, sie paßte schlecht zu diesem lieblichen Vormittag, und auch für einen, der die Gräber seiner Lieben besuchen will, war sie allzu trübselig. » (213). 
Cette fonction allégorique des saisons et du temps qu'il fait s'observe dans la plupart des nouvelles. Dans Der Wille zum Glück (1896), dans Der Tod (1897), comme dans Der Tod in Venedig (1912), la fin de l'été et l'arrivée de l'automne, puis de l'hiver sont incontestablement les signes annonciateurs de la mort des personnages. Mais, dans le même temps, l'attention aux phénomènes naturels et les postures qui y sont associées font l'objet d'une relecture critique qui tend à remettre en cause les valeurs affectives et le système de représentations qui les accompagnent.

Dans ce contexte, il convient de s'arrêter sur Der Tod (1897), une nouvelle qui a pu être considérée comme « l'une des plus faibles du jeune Thomas Mann »25. L'intérêt de ce récit pour notre propos est que les conditions atmosphériques sont indissociables ici de la fascination du personnage pour la mer, une mer qu'il vit essentiellement comme un spectacle, éprouvé du rivage et de la terre ferme, à l'image des poètes romantiques fascinés par le séjour sur les grèves (71). L'importance conférée à la mer et à son environnement est essentiellement fondée sur le sentiment du narrateur de vivre à la frontière de la terre et de l'eau, le long d'un rivage où « l'écume trouble » des eaux charriées par l'océan vient s'échouer (76). Fait notable, les thèmes de la mer et de la mort sont associés pour la première fois dans cette nouvelle 26 . Ce n'est donc pas une mer apprivoisée qui focalise l'attention, mais l'eau houleuse, « grise et vaste » (72), dont la contemplation suscite un faisceau d'émotions et de pensées où se mêlent le désir d'engloutissement et la peur de la vacuité, le rêve de fusion avec les éléments et la hantise de l'anéantissement :

[...] J'ai gardé l'œil rivé vers le dehors toute la nuit et j'avais l'impression que c'était cela la mort ou l'au-delà de la mort : là bas, dehors dans cette obscurité infinie, aux grondements sourds. Est-ce qu'une seule pensée ou intuition de moi subsistera là-bas [...] ?27

La détérioration du temps qu'il fait, le déchaînement progressif des vagues, de la pluie et du vent rythment la narration. Ils participent d'une dramaturgie de la catastrophe qui correspond à la situation extrême du personnage. La mousse trouble de l'eau et son obscurité infinie figurent à la fois l'abîme qui le guette et la force d'attraction des profondeurs. Ils représentent un territoire indécis des limites et des confins qui confronte le sujet avec quelque chose qui le dépasse et n'est pas lui. Support et instrument du questionnement existentiel du narrateur, la mer est à la

25. - Hans Rudolf Vaget, Thomas Mann. Kommentar zu sämtlichen Erzählungen, München, Winkler Verlag, 1984, 1986, p. 60.

26. - Roffmann constate qu'il s'agit du premier récit qui associe mer et mort, mais elle n'analyse pas le récit. A. Roffmann, « Keine freie Note mehr. », op. cit., p. 108.

27. - « Ich habe die ganze Nacht hinausgeblickt, und mich dünkte, so müsse der Tod sein oder das Nach-dem-Tode: dort drüben und draußen ein unendliches, dumpf brausendes Dunkel. Wird dort ein Gedanke, eine Ahnung von mir fortleben [...] (76). 
fois le miroir et le moteur de sa mélancolie ${ }^{28}$. Elle incarne une « expérience de l'éternité, du néant et de la mort, un rêve métaphysique ${ }^{29}$.

Cette mer n'offre guère de prise donc, et elle ne fait pas réellement l'objet d'une expérience directe, comme plus tard pour Tonio Kröger. Sa force d'évocation n'en est pas moins intense et témoigne, par rapport aux récits précédents, de l'émergence d'une conscience spécifique de la nature dont l'expression est liée à la pratique du journal intime, déterminé dans sa forme même par le passage des jours et des saisons. Ainsi, c'est l'enchaînement des notations au fil du temps et des pages, et l'écoute de soi propre au journal intime, qui semblent aiguiser l'esprit du diariste et le rendre sensible à la fuite et à la nécessité qui affectent les choses comme les idées :

Souvent, lorsque mes pensées s'étendent devant moi comme des eaux grises, qui semblent d'autant plus infinies qu'elles sont nébuleuses, je perçois quelque chose comme une cohérence des choses et crois reconnaître la vanité des concepts ${ }^{30}$.

La mer fait l'objet, dans ce texte, d'une expérience plus métaphysique que proprement esthétique : elle n'est pas encore cette surface de miroitements, de réfraction, de jeux d'optique et de sensations visuelles qui procure à Tonio Kröger plus tard le spectacle de l'illusion et de la mobilité des choses. Elle n'incarne pas non plus, comme pour Gustav Aschenbach, une promesse d'aventure ou un chemin vers l'exotisme, même si elle est associée, dans l'esprit du personnage, à son refus du conformisme bourgeois ${ }^{31}$. Cette expérience de la mer est aux antipodes de la vision thérapeuthique défendue par le docteur Gudehus qui recommande au comte les promenades à l'air libre pour le guérir de sa neurasthénie (74). Elle incarne des sentiments diffus et ambigus où se mêlent l'abandon de soi, physique et mental, et une forme de résistance aux forces extérieures incontrôlables qui affectent le monde naturel. Et, à ce titre, la fascination du narrateur pour le spectacle des vagues qui

28. - Cf. Daniel Linke, Ganz nordisch gestimmt... Jens Peter Jacobsens Einfluss auf das Frühwerk Thomas Manns, Marburg, Tectum Verlag, 2008. Linke a analysé l'importance du thème de la mélancolie dans les œuvres de jeunesse.

29. - « Das Meer ist keine Landschaft, es ist das Erlebnis der Ewigkeit, des Nichts und des Todes, ein metaphysischer Traum... ». Thomas Mann, « Lübeck als geistige Lebensform », op. cit., p. 394.

30. - « Ich habe die ganze Nacht hinausgeblickt, und mich dünkte, so müsse der Tod sein oder das Nach-dem-Tode: dort drüben und draußen ein unendliches, dumpf brausendes Dunkel. Wird dort ein Gedanke, eine Ahnung von mir fortleben [...] (75).

31. - Cf. A. Roffmann : «Die Meeresliebhaber im Werke Thomas Manns sind Deserteure des Bürgertums, die den Befehl zur Pflichterfüllung verweigern und stattdessen den Müßiggang am Meer genießen. » A. Roffmann, « Keine freie Note mehr. », op. cit., p. 248. 
viennent se briser à ses pieds et le bruit sourd du ressac (76) traduisent sa conscience de l'instabilité du moi.

Les historiens de la littérature ont souligné à ce propos l'influence de la métaphysique schopenhauerienne ${ }^{32}$. L'univers environnant, de fait, ne semble exister dans ce récit que par rapport et pour le sujet. Sa fascination pour les forces de la nature ne relève pas tant d'une ouverture au monde, que d'un repli du Moi sur lui-même.

La fin inopinée du récit qui ne s'achève pas sur la mort du narrateur, mais sur celle de la petite Asuncion, dont ce dernier se demande même s'il ne l'a pas provoquée (78), se lit toutefois comme un pied-de-nez ironique du destin et vient jeter une lumière trouble sur le sentiment métaphysique du personnage. Que la mort lui apparaisse de surcroît sous les traits d'un dentiste (77) dénie à celle-ci toute dimension transcendante et tourne en dérision la fascination décadente du comte pour la mort. Cette allégorie grotesque tend à démystifier la profondeur de son sentiment métaphysique en faisant apparaître son aspiration au néant comme une posture complaisante face à la mort. Elle contrecarre aussi la lecture symbolique que l'on pouvait faire du récit, en assimilant un peu trop rapidement le déchaînement des éléments à la mort annoncée du narrateur. On observe, qu'une fois encore, le romancier semble se jouer au dernier moment d'un système de représentations assimilant l'état de la nature à celui du sujet. Il introduit une distance entre la situation de l'homme et l'état du cosmos qui récuse une lecture symbolique univoque du monde.

La nouvelle Tonio Kröger (1903), l'« enfant littéraire préféré »33 de l'écrivain, est l'un des rares récits qui s'ouvre sur l'évocation du temps qu'il fait. Il est aussi le premier texte dans lequel l'opposition entre le Nord et le Sud est un principe structurant du récit. Kim Tschol-Za a mis en évidence que cette « espèce de grêle molle qui n'était ni de la glace ni de la neige » (243) qui tombe sur la ville au début du récit pouvait se lire comme un signe du déchirement de Tonio Kröger entre deux états, sa vie d'artiste et celle de bourgeois ${ }^{34}$. Dans Tristan (1903) aussi, « le dégel où tout s'amollit, goutte, barbote, coule » (355) reproduit sur un mode allégorique le renoncement de Frau Klöterjahn aux principes qui régissaient sa vie jusque-là. « La clarté laiteuse et blafarde du soleil d'hiver, caché par les couches de nuages » (243) évoque en ouverture de Tonio

32. - Ibid., p. 127. Voir aussi Hubert Ohl, Ethos und Spiel. Thomas Manns Frühwerk und die Wiener Moderne, Freiburg i. Br., Rombach, 1995, p. 63.

33. - Thomas Mann, « On myself », in : GW, Bd. XIII, p. 145.

34. - Kim Tschol-Za, Funktionen der Natur im Frühwerk Thomas Manns, Bonn, Rheinische Friedrich-Wilhelms-Universität, 1970, p. 150. 
Kröger un état d'indécision comparable. L'entre-deux qui caractérise le temps comme la couleur du ciel symbolise les déchirements intérieurs du personnage. Lorsqu'il reviendra bien des années plus tard dans sa ville natale, le brouillard, le vent humide et mordant, l'air gris et froid (283) seront, de la même facon, autant d'indicateurs de son arrachement aux certitudes rassurantes de sa vie passée. L'eau vague des nuages, des brumes et des brouillards transfigurent, en effet, les choses et les êtres. Ils transforment les apparences et signalent l'émergence d'un autre état, d'un autre monde.

Au-delà de cette dimension symbolique, on discerne toutefois, par rapport aux récits évoqués précédemment, un affinement de la perception du monde extérieur, qui marque aussi un enrichissement de la sensibilité. Dans cette nouvelle aussi, l'expérience de la mer est déterminante. Mais la mer Baltique n'est pas seulement l'objet d'une rêverie métaphysique qui « transporte au-delà de l'espace et du temps » (304). Elle est, cette fois, un lieu d'intensité des impressions et des émotions et, à ce titre, l'objet d'une expérience sensible de nature esthétique. Elle révèle la capacité d'émerveillement mais aussi d'attention de Kröger à ce qui se passe autour de lui. Et cela se traduit dans l'abondance et la richesse des verbes et des adjectifs qui la décrivent:

La mer dansait. Les vagues ne venaient pas à vous de manière ordonnée, rondes et égales, mais, jusqu'à l'horizon, sous une lumière pâle et vacillante, la mer était déchirée, fouettée, bouleversée; elle bondissait et léchait la nue de ses langues de géant, effilées comme des flammes, lançait en l'air, à côté d'abîmes bouillonnants, des figures déchiquetées et bizarres, et semblait éparpiller en un jeu fou, de toute la force de bras monstrueux, l'écume dans les airs ${ }^{35}$.

L'eau qui fascine Kröger n'est pas, comme dans le récit précédent, l'eau profonde et violente de la mer, de la pluie ou de l'orage qui assombrit le monde, qui promet l'engloutissement, mais l'eau tempétueuse qui bondit, assaille, submerge tout, en incarnant la force et la puissance du renouveau. La personnification dont elle fait l'objet tend à amplifier ses effets. Le mouvement des vagues figure un chaos qui n'est pas sans évoquer le chaos originel de la création. Les eaux de la mer semblent habitées de monstres informes, prêts à tout emporter. Le passage de la quiétude d'une mer plane, « à l'éclat blanchâtre et velouté » (303) à des images de submersion ou d'affrontement des éléments où « [1]es vagues

35. - « Das Meer tanzte. Nicht runde und gleichmäßige Wellen kamen in Ordnung daher, sondern weithin, in bleichem und flackerndem Licht, war die See zerrissen, zerpeitscht, zerwühlt, leckte und sprang in spitzen, flammenartigen Riesenzungen empor, warf neben schaumerfüllten Klüften zackige und unwahrscheinliche Gebilde auf und schien mit der Kraft ungeheurer Arme in tollem Spiel den Gischt in alle Lüfte zu schleudern. » (299). 
courbaient leurs têtes comme des taureaux qui s'apprêtent à donner des cornes et couraient rageusement contre la côte ${ }^{36}$ attestent le tumulte des aspirations contraires qui traversent le personnage. Elles symbolisent une instabilité régénératrice qui annonce la réorientation de son existence. «Absorbé », « enveloppé » par le fracas étourdissant du vent, « tout semble soudain devenir tranquille et chaud autour de lui. » ${ }^{37} \mathrm{Le}$ vent et la mer pénètrent et transforment son esprit. Le fracas des vagues, en définitive, le revivifie. Les descriptions de l'état du ciel et de l'eau témoignent ainsi de ce que Tonio Kröger, contrairement au narrateur de Der Tod, s'ouvre au monde qui l'entoure et de ce que ce monde semble, réciproquement, avoir prise aussi sur lui.

Nous évoquerons, pour finir Der Tod in Venedig (1912) pour rappeler que l'expérience de la mer s'y enrichit d'une dimension supplémentaire, en incarnant à la fois l'amour et la mort ${ }^{38}$. Ayant fait l'objet de nombreuses analyses sur lesquelles il ne s'agit pas de revenir, il nous importe essentiellement de souligner dans notre perspective que, contrairement à Tonio Kröger, Gustav Aschenbach développe une mythologie du soleil, de la mer et de la chaleur qui se déploie sur fond de refoulement de ses désirs. Comme le récit progresse, Aschenbach délaisse le rôle de l'écrivain austère et sentencieux, peu enclin à goûter en dilettante le chatoiement du monde des apparences, pour plonger dans un univers de sensations. En tournant le dos aux «étés pluvieux » passés en montagne (505), il part vers le soleil, assimilé, pour le meilleur et pour le pire, à l'ivresse dionysiaque des sens :

N'était-il pas écrit que le soleil détourne notre attention des choses intellectuelles vers les choses sensibles?

Il consumait maintenant en ivresse sentimentale, et dans une générosité imprévoyante, toute la vigueur que le soleil, le loisir et l'air marin lui fournissaient journellement ${ }^{39}$.

36. - « ¿ Die Wellen beugten die Köpfe wie Stiere, die die Hörner zum Stoße einlegen, und rannten wütend gegen den Strand. » (303).

37. - « Tonio Kröger stand in Wind und Brausen eingehüllt, versunken in dies ewige, schwere, betäubende Getöse [...] so schien es plötzlich ganz ruhig und warm um ihn her. » (303).

38. - Der Tod devait permettre à Thomas Mann de participer au concours de « la meilleure nouvelle dans laquelle la sexualité ne jouait aucun rôle. » Cf. Florian Lehmann, « Der Tod», in : A. Blödorn, F. Marx (dir.), Thomas Mann Handbuch, op. cit., p. 97.

39. - « Stand nicht geschrieben, daß die Sonne unsere Aufmerksamkeit von den intellekuellen auf die sinnlichen Dinge wendet ?» (553); « [...] so ließ er nun alles, was Sonne, Muße und Meerluft ihm an täglicher Kräftigung zuführten, hochherzig-unwirtschaftlich aufgehen in Rausch und Empfindung. » (558). 
Cette quête de lumière et de soleil qui conduit Aschenbach à plusieurs reprises durant la traversée vers Venise à guetter un éclaircissement du ciel (520), marque son renoncement à la respectabilité, aux allures solennelles et convenues, de même qu'à la musique réglée de son art. Ce faisant, il se laisse prendre dans les rêts d'une fiction du Sud, très marquée par la philosophie nietzschéenne, censée lui permettre de rompre avec sa vie passée. Soulignons, qu'à ce titre, le soleil est soumis à la référence antique et acquiert une valeur sacrée, inspirée du culte d'Helios dans la mythologie grecque (549), ses vertus se trouvant associées, du même coup, à celles de l'art.

Mais l'appel du Sud auquel répond Gustav Aschenbach ne prend pas la forme d'un voyage vers la fureur et le bonheur de vivre à l'italienne. Il se réalise dans une descente vers Venise, ville des lentes mélancolies, des eaux stagnantes de la Lagune et de la perte de soi dans le dédale des canaux. La mer étincelante qui fascine Tonio Kröger cède la place à la sensualité érotique de la liquidité et à « l'infini brumeux » (592) qui porte en lui la figure de l'irrévocable tout en étant aussi, à la fin du récit, invitation à un voyage sans retour.

Ce texte fait entendre des sons nouveaux, présents parfois, mais en latence, dans les récits précédents. De façon manifeste ici, les eaux putrides de Venise et l'air vicié par le choléra rendent présent ce qui est absent de la scène du langage et de la conscience du personnage, à savoir la dimension mortifère de ses affects et les connivences du désir avec la mort. Ainsi, l'ambivalence des significations du soleil, de l'eau et de la chaleur, de même que la confusion météorologique des saisons qui laisse s'épanouir un «faux été » au commencement du mois de mai (501), manifestent non seulement le dérèglement existentiel du personnage et ses errements, elles permettent de comprendre ce qu'il n'ose se dire à lui-même. Elles dessinent la configuration du non-dit et touchent aux territoires ténébreux de l'inconscient que Freud a commencé alors à baliser. La météorologie n'a donc, de ce point de vue, pas seulement une fonction mimétique de miroir des états du sujet. Elle fonctionne comme un révélateur des faux-semblants mortifères dans lesquels s'enferre le personnage.

\section{Conclusion}

Les notations atmosphériques et météorologiques qui parsèment les textes correspondent, de la sorte, à une mosaïque d'expériences et de gestes qui relèvent tout à la fois de la perception, de la construction imaginaire et de la manipulation. Le temps qu'il fait est prétexte, dans Gefallen, à un investissement émotif et symbolique que le narrateur tourne en dérision à la fin de son récit. Dans Der Tod, l'ambiance et le spectacle de la mer s'inscrivent au cœur d'un travail de l'imagination et 
de la pensée qui les soumettent à un travail d'interprétation en même temps que d'intériorisation. À la fois construction imaginaire et expérience vécue pour Tonio Kröger ou Gustav Aschenbach, l'état du ciel ou de la mer et les phénomènes naturels ne sont plus de simples objets de pensée ou d'émotion suscitant jeux d'association et errances de l'esprit. Ils sont des figures qui révèlent, comme en photographie, des aspirations latentes. Ainsi, un système de représentation fondé sur la symbolique des couleurs et des sons s'enrichit peu à peu sous l'effet de la capacité d'introspection que développent les personnages. La météo-sensibilité de ces derniers s'accroît avec leur capacité d'analyse et de perception des sensations. En même temps qu'ils s'enrichissent de significations nouvelles, les signes du temps se complexifient et, sous une apparente continuité, des mutations profondes s'opèrent. Les quelques exemples envisagés dans le cadre de cette étude témoignent de ce point de vue d'une évolution faite de recoupements et de décalages. En dépit des leitmotive, les personnages de Thomas Mann ne partagent pas tous le même ensemble de représentations et leurs rapports au temps ou aux saisons ne satisfont pas les mêmes désirs. Au fil du temps et des textes, la palette des pensées et des émotions qui les portent s'élargit, les quêtes, les plaisirs et les refoulements qu'ils manifestent se déplacent, les représentations convoquées font l'objet de dissolutions et de recompositions incessantes. Jamais pourtant l'observation du ciel ou l'attention portée aux conditions atmosphériques ne traduisent un sentiment durable d'appartenance à la nature. La représentation l'emporte régulièrement sur l'expérience directe, laquelle demeure, de ce fait, essentiellement visuelle. L'état du ciel, de la mer comme de la terre reste foncièrement tissé de métaphores et de symboles. Mais presque toujours est exhibé le pouvoir de l'écrivain qui n'hésite pas à retourner les systèmes de représentation et à se détacher des codes associés au temps qu'il fait ou à certains états de la nature. Pour lui, comme pour ces personnages, le ciel, le temps, les températures et l'état du monde environnant restent foncièrement liés aux joies et au désenchantement que procure le spectacle de l'illusion. Plus encore que leur fonction symbolique ou dramatique et leurs retentissements subjectifs, c'est bien cette relation de la météorologie avec les codes esthétiques et leur histoire qui semblent avoir attiré le styliste Thomas Mann. 
